die eine Rechtfertigung einer solchen Abgabe erlauben, sind ebenfalls vorhanden, sodass dem BEHG die Verfassungswidrigkeit nicht derart offensichtlich auf die Stirn geschrieben steht, wie es teilweise suggeriert wird. Erfreulich wäre es deshalb, wenn das Instrument aus der juristischen Perspektive offener aufgenommen würde und sich die Diskussion zu einzelnen Ausgestaltungsmerkmalen verschöbe, die noch rechtliche und ökonomische Fragen aufwerfen. ${ }^{101}$

Open Access. Dieser Artikel wird unter der Creative Commons Namensnennung 4.0 International Lizenz veröffentlicht, welche die Nutzung, Vervielfältigung, Bearbeitung, Verbreitung und Wiedergabe in jeglichem Medium und Format erlaubt, sofern Sie den/die ursprünglichen Autor(en) und die Quelle ordnungsgemäß nennen, einen Link zur Creative Commons Lizenz beifügen und angeben, ob Änderungen vorgenommen wurden.

Die in diesem Artikel enthaltenen Bilder und sonstiges Drittmaterial unterliegen ebenfalls der genannten Creative Commons Lizenz, so- fern sich aus der Abbildungslegende nichts anderes ergibt. Sofern das betreffende Material nicht unter der genannten Creative Commons Lizenz steht und die betreffende Handlung nicht nach gesetzlichen Vorschriften erlaubt ist, ist für die oben aufgeführten Weiterverwendungen des Materials die Einwilligung des jeweiligen Rechteinhabers einzuholen.

Weitere Details zur Lizenz entnehmen Sie bitte der Lizenzinformation auf http://creativecommons.org/licenses/by/4.0/deed.de.

Open Access funding enabled and organized by Projekt DEAL.

101) Angesprochen sind hiermit neben der Preisdiskussion z.B Fragen der Sozialverträglichkeit sowie der Zusammenführung mit dem EU-ETS, vgl. Edenhofer/Flachsland/Kalkuhl/Knopf/ Pahle, Bewertung des Klimapakets und nächste Schritte: $\mathrm{CO}_{2}^{-}$ Preis, sozialer Ausgleich, Europa, Monitoring, MCC-PIK, 2019.

\title{
https://doi.org/10.1007/s10357-021-3804-8 \\ Negativemissionstechnologien als neues Instrument der Klimapolitik: Charakterisiken und klimapolitische Hintergründe ${ }^{1}$
}

Till Markus, Romina Schaller, Erik Gawel, Klaas Korte

(C) Der/die Autor(en) 2021. Dieser Artikel ist eine Open-Access-Publikation.

Der ${ }^{1}$ Weltklimarat IPCC zeigt in seinem Sonderbericht „1,5 ${ }^{\circ} \mathrm{C}$ globale Erwärmung“" aus dem Jahr 2018, dass das Erreichen der im Pariser Übereinkommen vereinbarten Klimaziele nach wie vor möglich ist. Dies erfordere allerdings nicht nur einen grundlegenden Wandel unserer Lebens- und Wirtschaftsweise sowie die Reduktion von Treibhausgasemissionen auf Null (spätestens in der zweiten Hälfte dieses Jahrhunderts), sondern auch die dauerhafte Entnahme erheblicher Mengen von $\mathrm{CO}_{2}$ aus der Atmosphäre. Letzteres kann durch eine Reihe unterschiedlicher Verfahren und Technologien erreicht werden, sogenannte Negativemissionstechnologien (NETs). Der folgende zweigeteilte - Beitrag untersucht zentrale klimawissenschaftliche, -politische und -rechtliche Aspekte eines möglichen Einsatzes von Negativemissionstechnologien als Instrument der Klimapolitik. Hierzu werden aktuell diskutierte NETs vorgestellt und wesentliche Unterschiede $\mathrm{zu}$ anderen klimapolitischen Maßnahmen herausgearbeitet. Es zeigt sich, dass eine undifferenzierte Subsumtion von NETs unter den Begriff der Abschwächung (Mitigation) zu einer Verlagerung der Klimaschutzbemühungen führen kann, d.h. weg von Maßnahmen, die eine Vermeidung der Entstehung von Treibhausgasen bewirken, hin zu Verfahren

PD Dr. Till Markus, LL.M.,

Dr. Romina Schaller, LL.M. Eur.,

Department Umwelt- und Planungsrecht,

Helmholtz-Zentrum für Umweltforschung,

Leipzig, Deutschland

Prof. Dr. Erik Gawel,

Klaas Korte, Dipl.-Ök.,

Department Ökonomie,

Helmholtz-Zentrum für Umweltforschung,

Leipzig, Deutschland und Technologien, deren Beitrag zur Verwirklichung der Klimaschutzziele derzeit noch unsicher ist und die z. T. mit erheblichen Risiken behaftet sind.

\section{Einleitung und Hintergründe}

Die Vertragsparteien der Klimarahmenkonvention (KRK) einigten sich bereits im Jahr 1992 auf die Begrenzung der atmosphärischen Treibhausgaskonzentrationen. Zweck dieser Vereinbarung war und ist die Vermeidung einer ,gefährlichen anthropogenen Störung des Klimasystems".2 ${ }^{2}$ Ende 2015 wurde im Rahmen des Pariser Übereinkommens (PÜ) weitergehend vereinbart, dass der durch den Treibhauseffekt verursachte Anstieg der Erdtemperatur ,deutlich unter $2^{\circ} \mathrm{C}$ über dem vorindustriellen Niveau gehalten wird". 3 Weitere Anstrengungen sollen unternommen werden, um den Temperaturanstieg auf $1,5^{\circ} \mathrm{C}$ zu begrenzen. ${ }^{4}$ Zur Einhaltung der Erwärmungsobergrenze legt das PÜ darüber hinaus fest, dass der Scheitelpunkt der Treibhausgasemissionen ,so bald wie möglich“ erreicht werden

1) Hinweis: Der Beitrag ist im Rahmen der Helmholtz-Klima-Initiative (HI-CAM) erarbeitet worden. HI-CAM wurde mit Mitteln des Impuls- und Vernetzungsfonds der Helmholtz-Gemeinschaft (IVF) gefördert. Die Verantwortung für den Inhalt dieser Veröffentlichung liegt bei der Autorin und den Autoren.

2) Art. 2 S. 1 Rahmenübereinkommen der Vereinten Nationen über Klimaänderungen (Klimarahmenkonvention - KRK), BGBl. II, S. 1784 .

3) Art. 2 Abs. 1 lit. a PÜ. Zur Entwicklung der $2^{\circ}$ Celsius-Leitplanke, siehe Schlacke, Grenzwert oder Politikziel? Dogmatik und Legitimität der $2^{\circ}$ Celsius-Leitplanke, in Dilling/Markus, Ex Rerum Natura Ius? Recht aus der Natur der Sache, 2014, S. 93.

4) $1,5^{\circ} \mathrm{C}$, über dem vorindustriellen Niveau“, siehe Art. 2 Abs. 1 lit. a PÜ. 
soll, dass die Vertragsstaaten danach rasche Reduktionen herbeiführen, um in der zweiten Hälfte des Jahrhunderts ein Gleichgewicht zwischen Emissionen und ihrem Abbau durch Senken zu erzielen (netto-null) und dass sie ihre national festgelegten Beiträge (national determined contributions - NDCs) sukzessive steigern und nach , größtmöglicher Ambition" erklären und verwirklichen. ${ }^{5}$ Anders als noch das Kyoto-Protokoll sieht das PÜ indes keine eindeutigen Emissionsreduktionsziele für die Vertragsstaaten vor, sondern setzt auf ein System der freiwilligen Selbstverpflichtung, d.h. die Parteien legen ihre Reduktionsziele selber fest (,,nationally determined contributions" - NDCs). ${ }^{6}$

Allerdings ergibt sich aus der Festlegung der Temperaturziele einerseits und den Eigenschaften von $\mathrm{CO}_{2}$ als wichtigstem Treibhausgas andererseits durchaus ein sehr konkreter klimapolitischer Handlungsauftrag. Aufgrund der langen Verweildauer von $\mathrm{CO}_{2}$ in der Atmosphäre (mehrere hundert Jahre) nimmt seine dortige Konzentration stetig zu. Diese Zunahme erhöht den Treibhauseffekt und führt zu einem kontinuierlich Anstieg der globalen Mitteltemperatur. ${ }^{7}$ Im Hinblick auf die Erreichung des $2{ }^{\circ} \mathrm{C}-$ bzw. $1,5^{\circ} \mathrm{C}$-Ziels ist die „Lagerfunktion“ der Atmosphäre für $\mathrm{CO}_{2}$ demnach klar begrenzt (i. S. e. Gesamtbudgets). ${ }^{8}$ Laut dem Ende 2018 veröffentlichten IPCC-Sonderbericht „1,5 ${ }^{\circ} \mathrm{C}$ globale Erwärmung“ liegt die verbleibende Aufnahmekapazität im Hinblick auf die Erreichung des $1,5^{\circ}$ Ziels nur noch bei ca. 420 Gigatonnen $(\mathrm{Gt}) \mathrm{CO}_{2}{ }^{9}$ Bei gleichbleibenden Emissionsmengen wäre dieses Budget in etwas mehr als 10 Jahren erschöpft (für das $2^{\circ} \mathrm{C}$-Ziel blieben noch ungefähr 20 Jahre). ${ }^{10}$ Nehmen die Vertragsstaaten ihre Vereinbarungen ernst, dürfen ihre zukünftigen Emissionen in der Summe nicht dazu führen, dass die $1,5^{\circ} \mathrm{C}-$, wenigstens aber die $2^{\circ} \mathrm{C}$-Temperaturobergrenze überstiegen wird. ${ }^{11}$ Im Hinblick auf die Verteilung der verbleiben-

5) Art. 4 Abs. 1-3 Übereinkommen von Paris (PÜ), BGBl. II, S. 1082. „Netto-Emissionen sind definiert als durch anthropogene Entnahme reduzierte anthropogene Emissionen." Siehe IPCC, Sonderbericht $1,5^{\circ} \mathrm{C}$ globale Erwärmung, Zusammenfassung, 2018, S. 17. Netto-Null-Emissionen werden auch als Klimaneutralität bezeichnet. Siehe IPCC, Global Warming of $1,5^{\circ} \mathrm{C}, 2018$, S. 555.

6) Art. 3 S. 1 PÜ. Gemäß Art. 3 S. 2 PÜ sind einmal festgelegte Reduktionsziele in ihrem Ambitionsniveau fortlaufend zu steigern („,Progressionsgebot“). In diesem Systemwechsel erkannt viele Autoren ein Scheitern des internationale koordinierten Klimaschutz. Andere Autor`Innen interpretieren die Entwicklung positiver und hoffen auf Gestaltungs- und Steuerungskräfte jenseits der Staaten(gemeinschaft). Siehe z.B. Streck, Vertragsgestaltung im Wandel der internationalen Klimapolitik, ZUR 2019, 13; Franzius, Das Paris-Abkommen zum Klimaschutz, ZUR 2017, 515. Siehe auch Markus, Die Problemwirksamkeit des internationalen Klimaschutzrechts, ZaöRV 2016, 715.

7) Zur Geschichte und den für die Klima-Governance zentralen Aussagen der Klimawissenschaft siehe Simonis, Klimawandel Einführung in das transdisziplinäre Forschungsfeld, in: Simonis, Handbuch Globale Klimapolitik, 2017, S. 36.

8) Siehe IPCC (Fn. 5), Abschnitt C.1.3. Siehe auch den grundlegenden Überblick in Wissenschaftlicher Beirat der Bundesregierung Globale Umweltveränderungen ( $W B G U$ ), Kassensturz für den Weltklimavertrag - Der Budgetansatz, Sondergutachten, 2009, S. $9 \mathrm{ff}$.

9) IPCC (Fn. 5), Abschnitt C. 1.3.

10) Der IPCC bezieht sich dabei auf die globale mittlere Lufttemperatur in Bodennähe und eine 66-prozentige Wahrscheinlichkeit (mittleres Vertrauen). Siehe IPCC (Fn. 5), Abschnitt C.1.3. Siehe auch European Academies Science Advisory Council (EASAC), Negative emission technologies: What role in meeting the Paris Agreement targets?, 2018, S. 6.

11) Zur juristisch-dogmatischen Ausdeutung der beiden Ziele siehe z.B. Winter, Armando Carvalho et alii versus Europäische Union: Rechtsdogmatische und staatstheoretische Probleme einer Klimaklage vor dem Europäischen Gericht, ZUR 2019, 259 (262-263). Siehe auch Bodansky/Brunnée/Rajamani, International Climate Change Law, 2017, S. 210 f., 228f. Zur klimawissen- den Emissionen bzw. der anstehenden Reduktionslasten gibt das PÜ indes nur grobe Leitlinien vor. Zu beachten sind insoweit das Prinzip der ,geteilten aber unterschiedlichen Verantwortlichkeiten und jeweiligen Fähigkeiten“ sowie der „Grundsatz der Gerechtigkeit.“"12

Der IPCC-Bericht weist somit auf die Notwendigkeit eines grundlegenden Wandels unserer Lebens- und Produktionsbedingungen hin. Sollen die vereinbarten Temperaturziele noch erreicht werden, sind schnelle und weitreichende Systemübergänge in den Bereichen der Energiegewinnung und -nutzung, der Landwirtschaft, der Stadt- und Infrastrukturen sowie der Industrie erforderlich. Diese Systemübergänge müssen „,beispielslos bezüglich ihres Ausmaßes“ sein. ${ }^{13}$ Zur Illustration legt der IPCC vier unterschiedliche Minderungsstrategien vor, durch deren Umsetzung das $1,5^{\circ} \mathrm{C}$-Ziel erreicht werden könnte. ${ }^{14}$ Das hervorstechende Merkmal der vier Modellpfade ist, dass jeder von ihnen die dauerhafte Entnahme erheblicher Mengen $\mathrm{CO}_{2}$ aus der Atmosphäre vorsieht, also Negativemissionen. ${ }^{15}$ Laut IPCC seien solche Negativemissionen einerseits notwendig, um die auch nach einem Systemwandel verbleibenden Restemissionen auszugleichen, und andererseits, um einen möglichen ,emission overshoot“ wieder rückgängig zu machen (und die globale Erwärmung ggf. wieder auf 1,5 ${ }^{\circ} \mathrm{C}$ zurückzubringen). ${ }^{16}$ Der IPCC wählte diese Art der Darstellung, um den Handlungsrahmen für die Klimapolitik möglichst offen zu halten. ${ }^{17}$ Hinsichtlich des nötigen Entnahmeumfangs projizieren die Szenarien des IPCC eine Menge zwischen 100-1000 Gt $\mathrm{CO}_{2}$ bis Ende des 21. Jahrhunderts. ${ }^{18}$

Der Befund des IPCC zu Negativemissionen aus dem Jahr 2018 erregte bisher vor allem in der Wissenschaft und verschiedenen Ministerialverwaltungen Aufmerksamkeit. ${ }^{19}$ Erst langsam gelangt das Thema in das politische und öffentliche Bewusstsein. ${ }^{20}$

schaftlichen Perspektive siehe z.B. Robiou du Pont/Meinshausen, Warming assessment of the bottom-up Paris Agreement emissions pledges, Nature Communications 2018, 9:4810.

12) Siehe Art. 2 Abs. 2 und Art. 4 Abs. 1 PÜ. Zum Prinzip der modifizierten geteilten, aber unterschiedlichen Verantwortung im PÜ siehe Maljean-Dubois, The Paris Agreement: A new step in the gradual evolution of the differential treatment in the climate regime, RECIEL 2016 151; Voigt/Ferreira, Differentiation in the Paris Agreement, Clim. Law, 2016, 58.

13) IPCC (Fn. 5), Abschnitte C2 - C.2.7.

14) Zur kognitiv-normativen Dimension der Modelpfade siehe insbesondere Beck/Mahoney, The IPCC and the new map of science and politics, Wiley Interdiscip. Rev. Clim. Change 2018, 1.

15) IPCC (Fn. 5), 17 f., insbesondere Abschnitt C.3.

16) IPCC (Fn. 5), Abschnitt C. 3

17) Edenhofer/Kowarsch, Cartography of pathways: A new model for environmental policy assessments, Environ. Sci. Policy 2015, 56.

18) IPCC (Fn. 5), Abschnitt C.3. Zu den verschiedenen Annahmen in der Literatur siehe m. w. N. siehe EASAC (Fn. 10), S. 6.

19) Siehe vor allem The National Academy of Sciences Engineering, and Medicine (NAS), Negative emission technologies and reliable sequestration, 2019; EASAC (Fn. 10); Royal Society/Royal Society of Engineering, Carbon dioxide removal, 2018; Umweltbundesamt (UBA), Kurzposition zur Kohlendioxid-Entnahme aus der Atmosphäre - Carbon dioxide removal (sogenannte „Negativemissionen“), 2019.

20) Für Deutschland siehe z.B. den Evaluierungsbericht der Bundesregierung über die Anwendung des Kohlendioxid-Speicherungsgesetzes sowie die Erfahrungen zur CCS-Technologie vom 21.12.2018, BT-Drs. 19/6891, S. 15 f., sowie Sachverständigenrat für Umweltfragen (SRU), Für eine Entschlossene Umweltpolitik in Deutschland und Europa, 2020, S. 62 f. In der Presse wird das Thema bisher nur randständig angesprochen, siehe z.B. Fulterer, Die Corona-Krise spart mehr $\mathrm{CO}_{2}$ als je zuvor, der Klimaschutz profitiert trotzdem nicht, Neue Zürcher Zeitung v. 5. 5.2020. Die mangelnde öffentliche Diskussion problematisierend Anderson/Peters, The trouble with negative emissions, Science 2016, 182; Geden, The Paris Agreement and the inherent inconsistency of climate policy making, Wiley Interdiscip. Rev. Clim. Change 2016, 790 f. 
Tab. 1 Staaten, die NETs in ihre Klimastrategien integriert haben oder es planen (Erläuterungen der einzelnen NETs weiter unten)

\begin{tabular}{|c|c|c|}
\hline Land & Dokument (Jahr) & NETs \\
\hline Japan & The long-term strategy under the PA $(2019)^{21}$ & $\begin{array}{l}\text { - Aufforstung/Wiederaufforstung } \\
\text { - Direct Air Capture } \\
\text { - Blue Carbon }\end{array}$ \\
\hline Costa Rica & National Decarbonization Plan $(2019)^{22}$ & $\begin{array}{l}\text { - Aufforstung/Wiederaufforstung } \\
\text { - Erhöhung der Kohlenstoffbindung im Boden } \\
\text { - Blue Carbon }\end{array}$ \\
\hline Schweden & $\begin{array}{l}\text { Sweden's fourth Biennial Report under the UNFCCC } \\
(2019)^{23}\end{array}$ & $\begin{array}{l}\text { - Aufforstung/Wiederaufforstung } \\
\text { - BECCS } \\
\text { - Erhöhung der Kohlenstoffbindung im Boden }\end{array}$ \\
\hline Dänemark & $\begin{array}{l}\text { Denmark's integrated National Energy and Climate Plan } \\
(2019)^{24}\end{array}$ & - Aufforstung \\
\hline Vereinigtes Königreich & The Clean Growth Strategy $(2017)^{25}$ & $\begin{array}{l}\text { - Aufforstung/Wiederaufforstung } \\
\text { - BECCS } \\
\text { - Erhöhung der Kohlenstoffbindung im Boden } \\
\text { - DAC } \\
\text { - Blue Carbon } \\
\text { - Beschleunigte Verwitterung an Land }\end{array}$ \\
\hline Kanada & $\begin{array}{l}\text { Canada's mid-century long-term low-greenhouse gas } \\
\text { development strategy }(2016)^{26}\end{array}$ & $\begin{array}{l}\text { - BECCS } \\
\text { - Aufforstung }\end{array}$ \\
\hline Portugal & Roadmap for carbon neutrality 2050 (RNC2050) (2019) ${ }^{27}$ & $\begin{array}{l}\text { - Aufforstung } \\
\text { - BECCS }\end{array}$ \\
\hline
\end{tabular}

Die Reaktionen und Wertungen unterscheiden sich z. T. recht stark. Idealtypisch lassen sich drei Argumentationsstränge identifizieren. ${ }^{28}$ Einerseits erweckt das Vorgehen des IPCC, d.h. die Einbeziehung signifikanter Mengen von Negativemissionen in seine Modellpfade, Hoffnungen auf effektive Mittel zur Bekämpfung des Klimawandels. ${ }^{29}$ Die Erzeugung von Negativemissionen entweder durch die Beschleunigung oder Erweiterung natürlicher Prozesse oder durch den Einsatz technischer Verfahren (zusammenfassend Negative Emission Technologies - NETs genannt), seien dringend oder sogar zwingend nötig, da die NDCs der Staaten im Hinblick auf die Erreichung der Temperaturobergrenzen immer noch viel zu niedrig ausfielen. ${ }^{30} \mathrm{Po}-$ litik und Recht müssten der Wissenschaft und Wirtschaft daher klare Anreize und Planungssicherheit bieten, um die Erforschung und Entwicklung dieser Technologien voranzutreiben. Nur so könne die volle Gestaltungskraft des Marktes für die Lösung des Klimawandels nutzbar gemacht werden (Grundmotiv: „Humanity's best hope is to promise that the next crop of billionaires will be those who figure out low-cost carbon dioxide removal" ${ }^{31}$ Eine skeptisch-kritische Argumentationskette hebt demgegenüber darauf $a b$, dass viele der Methoden zur Erzeugung von Negativemissionen nicht die eigentlichen Ursachen des Problems adressierten, sondern lediglich dessen Folgen. ${ }^{32}$ Derartige Ansätze erforderten z. T. sogar weitere belastende Eingriffe in die Natur, was in den meisten Fällen lediglich zu einer Verlagerung des Klimaproblems führe. Außerdem seien die $\mathrm{CO}_{2}$-Entnahmepotenziale, mögliche negative Folgen sowie die Kosten vieler NET-Techniken derzeit nur schwer abschätzbar. Darüber hinaus sei die langfristige Durchführung großskaliger, evtl. grenzüberschreitender Maßnahmen aus akzeptanz- und geopolitscher Perspektive überaus voraussetzungsreich. Ein überoptimistisches Vertrauen in NETs setze weiterhin einen Anreiz, Mitigationsanstrengungen zu reduzieren (Grundmotiv: „NETs are end-of-pipe-technologies, risky, and create moral hazards"). ${ }^{33}$ Der dritte, skeptischoptimistische Strang priorisiert eindeutig die proaktive Begrenzung von Treibhausgasen und benennt kritisch die vielen offenen Fragen hinsichtlich der Entwicklungs- und Einsatzmöglichkeiten von NETs. Dennoch wird die Möglichkeit ihrer ergänzenden, wenn auch begrenzten Funk- tion als Instrument der Klimapolitik nicht grundsätzlich ausgeschlossen. Auch wenn NETs kein Allheilmittel seien, können sie doch unter bestimmten Voraussetzungen einen Beitrag zur Vermeidung insbesondere eines gefährlichen Klimawandels leisten. Es gelte daher, die Kosten, Risiken, Wirksamkeitsfaktoren von NETs weiter zu erforschen. Insoweit sei allerdings durchaus Eile geboten, denn die Ent-

21) The Government of Japan, The long-term strategy under the Paris Agreement, 2019, S. 64f; S. 79-81.

22) The Government of Costa Rica, National Decarbonization Plan, 2019, S. 60.

23) Swedish Ministry of the Environment, Sweden's fourth Biennial Report under the UNFCCC, 2019, S. 42.

24) Danish Ministry of Climate, Energy and Utilities, Denmark's integrated National Energy and Climate Plan, 2019, S. 8, S. 30

25) Der Begriff NETs wird nicht verwendet, sondern GGR-Technologien (Entnahme von Treibhausgasen). The Government of the United Kingdom, The Clean Growth Strategy, 2017, S. 57, S. 69 f., S. 115.

26) The Government of Canada, Canada's mid-century long-term low-greenhouse gas development strategy, 2016, S. 57, S. 61.

27) Portuguese Ministry of the Environment and Energy Transition, Roadmap for carbon neutrality 2050 (RNC2050), 2019, S. 41, S. 63.

28) Innerhalb der hier skizzierten Argumentationsstränge gibt es z. T. starke Wertungs- und Einschätzungsunterschiede zu einzelnen Fragen und Aspekten. Die hier folgenden Verweise sind lediglich exemplarisch, d.h. die darin referenzierten Quellen vertreten nicht in toto die hier typisierten Argumente.

29) Siehe exemplarisch für diese Position Krupp/Keohane/Pooley, Less than zero - Can carbon-removal curb climate change?, Foreign Aff. 2019, 142

30) So z.B. Haszeldine et al., Negative emissions technologies and carbon capture and storage to achieve the Paris Agreement commitments, Phil. Trans. R. Soc. A 2018, 20160447, 13.

31) Krupp/Keohane/Pooley, (Fn. 22), 152.

32) Siehe exemplarisch Martin, The dubious promise of bioenergy plus carbon capture, Stand 8. Januar 2016, abrufbar unter: https://www.technologyreview.com/2016/01/08/247100/thedubious-promise-of-bioenergy-plus-carbon-capture/; Ginzky/ Ruddigkeit, Geo-Engineering: doch eine Antwort auf die Klimakrise?, ZUR 2019, 449.

33) Siehe auch die verschiedenen Einträge unter http://www.geo engineeringmonitor.org/. 
wicklung und Effektuierung von NETs benötige mehrere Jahre bis Jahrzehnte, ein ad hoc Einsatz angesichts einer drohenden Klimakatastrophe würde das Risiko unerwünschter Nebeneffekte deutlich erhöhen (Grundmotiv: „,we need all possible tools“). ${ }^{34}$ Diesem letzten Argumentationsstrang folgt eine zusehends wachsende Zahl von Staaten, die ihre nationalen Klimastrategien vor allem in den letzten beiden Jahren durch verschiedenen NETs ergänzt haben (nur Kanada und das Vereinigte Königreich hatten bereits vorher angekündigt, NETs einsetzen zu wollen). In der deutschen Klimapolitik spielen NETs derzeit nur eine untergeordnete Rolle. Analysiert und diskutiert werden aktuell aber sowohl die Entnahmepotenziale einer nachhaltigen land- und forstwirtschaftlichen Flächenbewirtschaftung als auch der Kohlenstoffabscheidung und Einlagerung (Carbon Capture and Storage - CCS) sowie verschiedener Abscheidungs- und Verwertungsansätze (Carbon Capture and Use - CCU). ${ }^{35}$

Die wachsende Bedeutung, die der IPCC den NETs bei der Umsetzung des PÜ zuweist, die große Diskrepanz zwischen seinen Modellen und den vielen offenen Fragen hinsichtlich ihrer Realisierbarkeit, die zunehmende Offenheit verschiedener Staaten gegenüber NETs als Instrument der Klimapolitik sowie die immer noch defizitären nationalen NDCs im Rahmen des PÜ bieten hinreichende Anlässe, sich den zentralen politischen, rechtlichen und ökonomischen Fragen zuzuwenden, die mit der Erforschung und dem möglichen Einsatz von NETs zusammenhängen. ${ }^{36}$

Dieser Beitrag will eine rechtliche und ökonomische Orientierung hinsichtlich der Potentiale von NETs als mögliches Instrument der Klimapolitik geben. Hierzu soll untersucht werden, inwieweit sie im Rahmen des internationalen Klimaregimes bereits eingesetzt und geregelt werden. Untersuchungsleitend ist die These, dass die zunehmende Bedeutung von NETs als Instrument der Klimapolitik, ihre klare analytische, begriffliche und dogmatische Abgrenzung von Maßnahmen erfordert, die bereits die Entstehung anthropogener Treibhausgase verhindern. Weiterhin ist ihre Unterscheidung von Methoden der Anpassung sowie des Solar Radiation Managements bedeutsam. Würden NETs in dem vom IPCC angedachten Umfang zum Einsatz kommen, ergäbe sich hieraus eine völlig neue Sachlage, die es im Klimaregime abzubilden gälte. Das Klimaregime ist insoweit entwicklungsoffen angelegt, d. h. es lässt eine differenzierte Behandlung der unterschiedlichen Instrumente der Klimapolitik zu und fordert sie sogar ein. Das lässt sich insbesondere auch aus den bisherigen Erfahrungen mit Senken im Klimaregime schließen. Die Untersuchung der Maßnahmen zur Umsetzung der Senkenfunktion im Klimaregime gibt darüber hinaus wichtige Hinweise auf politische, rechtliche und ökonomische Herausforderungen des möglichen Einsatzes von NETs als Instrument der Klimapolitik.

In diesem ersten Teil des Beitrags werden die aktuell diskutierten NETs überblicksartig vorgestellt (2.1). Wirkungen und mögliche Nebenwirkungen sollen angesprochen werden (2.2). Weitergehend sollen ihre grundlegenden strukturellen Unterschiede zu den Maßnahmen der Begrenzung von Treibhausgasen, zur Anpassung sowie zum SRM herausgearbeitet werden (2.4). Der erste Teil des Beitrags endet mit einem Zwischenergebnis (3.). Im zweiten Teil gilt es, die verschiedenen Techniken im internationalen Klimaregime zu verorten. Insbesondere soll ihre potentielle Funktion als Senke im Sinne der KRK untersucht werden. Hierzu erfolgt ein Rückblick auf die bisherige Praxis der Nutzung von Senken. Darauf aufbauend können einige Rückschlüsse hinsichtlich der zentralen Rechtsfragen gezogen werden, die sich im Zusammenhang mit einer möglichen Entwicklung und Anwendung von NETs als Instrumente der Klimapolitik stellen. Anschließend wird eine rechtliche und ökonomische Forschungsperspektive entwickelt. Der Beitrag endet mit einem Fazit.

\section{Charakteristiken von Negativemissionstechnologien}

In den folgenden Unterabschnitten werden verschiedene NETs vorgestellt. Dabei wird der derzeitige Kenntnisstand hinsichtlich ihrer Potentiale zur Entnahme von Treibhausgasen aus der Atmosphäre zusammengefasst sowie ihrer möglichen Nebenwirkungen aufgezeigt. Weitergehend erfolgt eine analytische, funktionale und begriffliche $\mathrm{Ab}$ grenzung zu anderen Klimaschutzmaßnahmen.

\subsection{Typen}

NETs umfassen eine Reihe sehr unterschiedlicher Methoden zur bewussten Entnahme von Treibhausgasen aus der Atmosphäre. ${ }^{37}$ Geht es ausschließlich um die Entnahme von $\mathrm{CO}_{2}$ als wichtigstem Treibhausgas, werden die Begriffe NETs und Carbon Dioxide Removal (CDR) häufig synonym verwendet. ${ }^{38}$ Damit eine Entnahme langfristig wirksam ist, müssen die Gase im Boden, im Meer (bzw. im Meeresboden) oder in künstlichen Speichern eingelagert werden (storage/sequestration). Auch ist eine Verwertung grundsätzlich vorstellbar, bei der die entnommenen Treibhausgase stofflich gebunden bleiben und (vorerst) nicht wieder in die Atmosphäre gelangen (usage). ${ }^{39}$

Die aktuell diskutierten NETs können anhand unterschiedlicher Distinktionsmerkmale klassifiziert werden: ${ }^{40}$ Einige NETs nutzen die Beschleunigung und Ausweitung natürlicher Prozesse zur Kohlenstoffbindung, andere sind technologiebasiert. ${ }^{41}$ Einige Methoden lagern Treibhaus-

34) Dies ist im Grundsatz die Lesart des IPCC. Siehe aber auch EASAC (Fn. 10). Etwas optimistischer The National Academy of Sciences, Engineering, and Medicine, Negative Emission Technologies and Reliable Sequestration: A Research Agenda, 2019. Siehe auch Edenhofer/Jakob, Klimapolitik, 2019, S. 54-56. In der Rechtswissenschaft exemplarisch Lin, Negative emissions after Paris, Ecol. L. Q. 2019, 533; Gerard, Introduction and overview, in: Gerard/Hester, Climate Engineering and the Law, 2018, S. 1.

35) $U B A$, Wege in eine ressourcenschonende Treibhausgasneutralität - RESCUE-Studie, 2019, S. 32. Siehe BT- Drs. 19/6891, S. 15 f.; $S R U$ (Fn. 20), S. 62 f.

36) Im Dezember 2019 zeichnete sich ab, dass es trotz der NDCs aller Vertragsparteien des PÜ in besten Fall zu einer globalen Erwärmung von $2,8^{\circ} \mathrm{C}$ gegen Ende des Jahrhunderts kommen wird. Siehe Climate Action Tracker, Warming Projections Global Update December, 2019, S. 1.

37) Die Entnahme erfolgt zusätzlich zu der Entnahme, die sich bereits aus natürlichen Prozessen erfolgen würden. Siehe z. B. IPCC (Fn. 5), S. 554; Minx et al., Negative emissions - Part 1: Research landscape and synthesis, Environ. Res. Lett. 2018, 063001.

38) NETs adressieren grundsätzlich auch andere Klimagase als $\mathrm{CO}_{2}$. Der IPCC $1,5^{\circ} \mathrm{C}$-Bericht bezieht sich allerdings vor allem auf das Carbon Dioxide Removal, siehe IPCC (Fn. 5), Abschnitt C. 3f. Zu Methan siehe aber beispielsweise Stolaroff et al., Review of methane mitigation technologies with application to rapid release of methane from the Arctic, Environ. Sci. Technol. 2012, 6455; Secretariat of the Convention on Biological Diversity, Update on climate geoengineering in relation to the Convention on Biological Diversity, 2016, S. 68.

39) Wichtig ist insoweit, dass das Gas bei der Weiterverwendung nicht in die Atmosphäre emittiert wird.

40) Pires, Negative emissions technologies: A complementary solution for climate change mitigation, 2019, S. $502 \mathrm{ff}$; EASAC (Fn. 10), S. 7 f.; Minx et al. (Fn. 37); McGlashan et al., Negative emissions technologies, 2012, S. 2; de Richter/Caillol/Ming, Geoengineering: Sunlight reflection methods and negative emissions technologies for greenhouse gas removal, in: Letcher, Managing Global Warming, 2019, S. $593 \mathrm{f}$; Psarras et al., Slicing the pie: How big could carbon dioxide removal be?, Wiley Interdiscip. Rev. Energy Environ. 2017, e253, 5 f.

41) Unter dem Begriff der Negativemissionstechnologie werden im Diskurs auch naturbasierte Ansätze gefasst, wenngleich diese keine Technologie im engeren Sinne darstellen. Siehe Minx et al. (Fn. 37), S. 5. 
gase terrestrisch ein (z.B. im Boden), andere im Meer oder im Meeresboden. Einige Techniken nutzen biologische Prozesse zur Entnahme (insb. Photosynthese), andere chemische oder physikalische Verfahren.

Die größte Aufmerksamkeit unter den derzeit diskutierten naturbasiert-terrestrischen NETs erhält das sogenannte „Bioenergy with Carbon Capture and Storage" (BECCS). ${ }^{42}$ Grund hierfür ist wohl die mögliche kommerzielle Verwertbarkeit der im Rahmen dieses Verfahrens gewonnenen Energie. ${ }^{43}$ Bei BECCS wird großskalig Biomasse zur Energieerzeugung genutzt und das dabei entstehende $\mathrm{CO}_{2}$ abgeschieden und unterirdisch eingelagert. ${ }^{44}$ Die Biomasse kann aus speziell dafür angebauten Energiepflanzen oder aus anderen forst- und landwirtschaftlichen Quellen stammen. ${ }^{45}$ Eine weitere Möglichkeit, Negativemissionen zu erzeugen, bieten die Aufforstung und die Wiederaufforstung, also die großskalige Neu- oder Wiederbewaldung ehemals bewaldeter Flächen. ${ }^{46}$ Der Anbau von Bäumen bindet $\mathrm{CO}_{2}$ aus der Atmosphäre, die so entstehenden Wälder bilden den Kohlenstoffspeicher. ${ }^{47}$ Daneben wird auch die Nutzung von Biokohle zur Erzeugung von Negativemissionen diskutiert (Biochar). Dies beinhaltet das ,Verkohlen" von Biomasse (durch thermochemische Konversion oder hydrothermale Karbonisierung) sowie das Einbringen der Kohle in Böden zur Einlagerung des darin enthaltenen $\mathrm{CO}_{2} \cdot{ }^{48}$ Ein weiteres Verfahren ist die beschleunigte Verwitterung. Dabei wird der natürliche Zerfallsprozess bestimmter Gesteine künstlich vorangetrieben und dadurch $\mathrm{CO}_{2}$ chemisch oder physikalisch gebunden. Dies kann z. B. durch das Zermahlen von kalzium- und magnesiumhaltigem Silikatgestein geschehen, das dann über große Flächen von Ackerland verteilt wird. Weitergehend wird erörtert, inwieweit eine veränderte Bewirtschaftungspraxis von Böden deren Kohlenstoffbindung erhöhen kann. ${ }^{49}$ Im Kern geht es dabei einerseits um die Stärkung von Nutzungspraktiken, die den Eintrag von $\mathrm{CO}_{2}$ in Böden erweitern, z.B. durch die Wiedervernässung und nachhaltige Bewirtschaftung von Mooren (Paludikultur), eine generell verbesserte landwirtschaftliche Praxis oder ein systematisches Feuer-Management in Wäldern sowie andererseits um die Vermeidung solcher Aktivitäten, die das Entweichen von $\mathrm{CO}_{2}$ aus Böden begünstigen (z.B. die Umwandlung von Grasland). ${ }^{50}$

Als meeresbasierte NETs werden vor allem das sogenannte Blue Carbon, die Alkalisierung der Meere und die Ozeandüngung, thematisiert. Blue Carbon umfasst primär solche Techniken, bei denen $\mathrm{CO}_{2}$ in Meeres- und Küstenökosystemen gespeichert wird, insbesondere durch Seegraswiesen, Salzwiesen, und Mangrovenwälder. Die Erweiterung und der Schutz dieser Gebiete können einen Beitrag zur Kohlenstoffentnahme und -speicherung leisten. ${ }^{51}$ Die Alkalisierung der Meere zielt wiederum darauf $a b$, durch die Erhöhung der Alkalinität, z. B. durch $\mathrm{Ca}(\mathrm{OH})_{2}$, deren Aufnahmekapazität für $\mathrm{CO}_{2}$ erweitern. ${ }^{52}$ Zuletzt geht es bei der Ozeandüngung darum, bestimmten nährstoffarmen Meeresregionen (in aller Regel küstenfern) „Dünger“ zuzuführen (z.B. Eisen), um so Algenwachstum anzuregen. Durch das Algenwachstum wird $\mathrm{CO}_{2}$ aus der Atmosphäre gebunden. Nach ihrem Ableben sinken die Algen auf den Meeresgrund und lagern dort das $\mathrm{CO}_{2}$ ein..$^{53}$

Neben diesen Verfahren, die allesamt auf die Veränderung oder Beschleunigung des natürlichen Karbonkreislaufs abzielen, werden zunehmend technologiebasierte Verfahren zum Herausfiltern von Treibhausgasen aus der Atmosphäre erforscht (Direct Air Capture - DAC). ${ }^{54} \mathrm{CO}_{2}$ wird hier vor allem unter Einsatz chemischer Bindemittel aus der Umgebungsluft eingefangen, komprimiert, unterirdisch gelagert (Direct Air Carbon Capture and Storage -DACCS) oder einer weiteren Nutzung zugeführt (DACCU). ${ }^{55}$ Aus einer Weiterverwendung resultieren Negativemissionen allerdings nur in dem Umfang, in dem der aus der Atmosphäre entnommene Kohlenstoff langfristig gebunden bleibt. Die
Dauer der Einlagerung hängt im Wesentlichen von der Lebensdauer der Produkte ab. Auf lange Sicht ist dieser Ansatz daher im besten Fall klimaneutral, weshalb man auch von einem ,circular carbon approach" spricht. ${ }^{56}$

An dieser Stelle ist noch auf das im Zusammenhang mit Negativemissionen (erneut) viel diskutierte Carbon Capture and Storage (CCS) einzugehen. ${ }^{57}$ Beim CCS, so wie es bisher konzipiert wurde, wird die Bildung von $\mathrm{CO}_{2}$ nicht im eigentlichen Sinne vermieden. $\mathrm{CO}_{2}$ wird während der Energieerzeugung oder im Rahmen von industriellen Produktionsprozessen abgeschieden und unterirdisch eingelagert. Im Ergebnis wird also lediglich die Übergabe von Treibhausgasen an die Atmosphäre verhindert, i. S.e. Zurückhaltung oder Retention. Strukturell liegt CCS den NETs damit näher als den Ansätzen, die bereits die Entstehung von Treibhausgase verhindern. Hinzu kommt, dass einige NETs das CCS zwingend voraussetzen: Es kann kein BECCS und kein DACCS ohne CCS geben. ${ }^{58}$ Auch

42) BECCS nimmt eine zentrale Rolle in den Minderungsszenarien des IPCC ein, siehe IPCC (Fn. 5), Abschnitt C. (S. 17f.). Siehe auch Creutzig et al., The mutual dependence of negative emission technologies and energy systems, Energy Environ. Sci. 2019, 1807.

43) Anderson/Peters, The trouble with negative emissions, Science 2016, 182, 183.

44) IPCC (Fn. 5) S. 543; The Royal Society/Royal Academy of Engineering, Greenhouse Gas Removal, 2018, S. 39; Kemper, Biomass and carbon dioxide capture and storage, Int. J. Greenh. Gas Control 2015, 401.

45) The Royal Society/Royal Academy of Engineering (Fn. 44), S. 39

46) Aufforstung ist die direkte Umwandlung von Flächen in Waldgebiete, die seit mindestens 50 Jahren nicht mehr bewaldet wurden. Wiederaufforstung wird verstanden als die Wiederbewaldung kürzlich abgeholzter Flächen. United Nations Framework Convention on Climate Change (UNFCCC), Report of the Conference of the Parties serving as the meeting of the Parties to the Kyoto Protocol on its first session, held at Montreal from 28 November to 10 December 2005 (FCCC/KP/CMP/2005/8/Add.3), Decision 16/CMP.1, Annex; UNFCCC, CDM Methodology Booklet, 2019, S. 39.

47) Siehe z. B. Dixon et al., Carbon pools and flux of global forest ecosystems, Science 1994, 185, 188; Pan et al., A large and persistent carbon sink in the world's forests, Science 2011, 988, 989.

48) Fuss et al., Negative emissions - Part 2: Costs, potentials and side effects, Environ. Res. Lett. 2018, 063002, 30; Pires (Fn. 40), 511; $U B A$ (Fn. 19), S. 5.

49) The Royal Society/Royal Academy of Engineering (Fn. 44), S. 32.

50) Siehe z.B. die Studie von Fargione et al., Natural climate solutions for the United States, Sci. Adv. 2018, eaat1869.

51) IPCC (Fn. 5), S. 543; Herr/Landis, Coastal blue carbon ecosystems, 2016, S. 8 f.; Vierros, Communities and blue carbon: The role of traditional management systems in providing benefits for carbon storage, biodiversity conservation and Livelihoods, Clim. Change 2017, 89; Macreadie et al., The future of blue carbon science, Nat. Commun. 2019, 1.

52) Fuss et al. (Fn. 48), 21.

53) Smetacek/Naqvi, The next generation of iron fertilization experiments in the Southern Ocean, Philos. Trans. R. Soc. A. 2008; Boyd et al., Mesoscale iron enrichment experiments 1993-2005: Synthesis and future directions, Science 2007, 612; Buesseler et al., Ocean iron fertilization - Moving forward in a sea of uncertainty, Science 2008, 162; siehe auch die interdisziplinäre Perspektive Güssow et al., Ocean iron fertilization: Why further research is needed, Mar. Policy 2010, 911.

54) Dittmeyer et al., Crowd oil not crude oil, Nat. Commun. 2019, 1818; Keith et al., A process for capturing $\mathrm{CO}_{2}$ from the atmosphere, Joule 2018, 1573.

55) Pires (Fn. 40), 504; Psarras et al. (Fn. 40), 8; NAS (Fn. 19), S. 196; The Royal Society/Royal Academy of Engineering (Fn. 44), S. 59.

56) Hepburn et al., The technological and economic prospects for $\mathrm{CO}_{2}$ utilization and removal, Nature 2019, 87, 88f.; Dittmeyer et al. (Fn. 54), 2.

57) Dieser enge Zusammenhang wird auch im Sonderbericht des IPCC aus 2018 erkennbar. Siehe hierzu IPCC (Fn. 5), Abschnitt C. (S. $17 \mathrm{f}$.).

58) Haszeldine et al. (Fn. 23), 3. 
unabhängig von der Entwicklung um NETs wird CCS seit einem IPCC-Bericht aus dem Jahr 2005 als Baustein der Klimapolitik diskutiert. ${ }^{59}$ Die Diskussionen haben in jüngster Zeit wieder zugenommen; ein Effekt der sich voraussichtlich aufgrund der Debatte um NETs noch verstärken wird. ${ }^{60}$

\subsection{Zur Charakteristik von NETs:}

Potenziale und Einsatzvoraussetzungen

Die Potentiale der einzelnen NETs zur Entnahme und dauerhaften Einlagerung von Treibhausgasen werden in der Wissenschaft derzeit z. T. recht unterschiedlich bewertet. Die ganz überwiegende Anzahl der Wissenschaftler geht allerdings davon aus, dass NETs keine gleichwertige Alternative $\mathrm{zu}$ ambitionierten Vermeidungsstrategien darstellt. ${ }^{61}$ Weitergehend scheint ein Konsens dahingehend zu bestehen, dass das Potential der einzelnen Ansätze derzeit von verschiedenen, unsicheren Voraussetzungen abhängt. Überwiegend erfordern NETs ein langfristiges und großskaliges Engagement hinsichtlich Forschung, Entwicklung und Anwendung. Sie bieten also keine schnelle Lösung für das Klimaproblem. Bis auf die Aufforstung- und Wiederaufforstung befinden sich die meisten NETs noch in einem frühen Entwicklungsstadium. Dies spiegelt sich nicht $z u-$ letzt in den aktuell erheblich divergierenden Einschätzungen hinsichtlich der Potentiale einzelner NETs zur Entnahme von $\mathrm{CO}_{2}$ aus der Atmosphäre wider.

Ein wichtiger Teilaspekt der Effektivität von NETs ist die Möglichkeit ihres dauerhaften und weiträumigen Einsatzes. So setzen z.B. die Aufforstung- und Wiederaufforstung sowie BECCS langfristig und groß angelegte Bewirtschaftungsmaßnahmen von Wäldern oder den Anbau von Bioenergiepflanzen voraus. Einerseits kann die Dauer der Einlagerung bei diesen naturbasierten Ansätzen durch natürliche Faktoren stark eingeschränkt werden (z.B. durch Trockenperioden, Brände und Krankheiten). ${ }^{62}$ Andererseits ist die Verwirklichung umweltpolitischer Infrastrukturprojekte in ähnlicher Größenordnung in den letzten Jahren in verschiedenen Staaten auf große Akzeptanzprobleme gestoßen. Beispielhaft können die Proteste gegen verschiedene Elemente der Energiewende in Deutschland genannt werden, insbesondere im Zuge des Netz- und Wind-

59) IPCC, Carbon dioxide capture and storage, 2005.

60) Siehe z.B. International Energy Agency, Transforming industry through CCUS, 2019; Wennerstein/Sun/Li, The future potential of carbon capture and storage in climate change mitigation, J. Clean. Prod. 2015, 724; EASAC, Carbon capture and storage in Europe, 2013. Für Deutschland nach wie vor kritisch jüngst $U B A$ (Fn. 19), S. 7. Siehe auch BT- Drs. 19/6891, S. 15 f.

61) Siehe EASAC (Fn. 10), S. 1 f.; Smith et al., Biophysical and economic limits to negative $\mathrm{CO}_{2}$ emissions, Nat. Clim. Chang. 2015; Instruktiv hinsichtlich der natürlichen, nicht CCS-gestützten Ansätze zuletzt Fargione et al. (Fn. 50).

62) Siehe z.B. Wiliamson, Emissions reduction: Scrutinize $\mathrm{CO}_{2}-\mathrm{re}-$ moval methods, Nature 2016, 153, 154.

63) Die für den Bereich des Minderungspotenzials im Jahr 2050 verwendeten Daten stammen überwiegend aus Studien und Berichten von Fuss et al. (Fn. 48), IPCC (Fn. 5); NAS (Fn. 19); EASAC (Fn. 10); Minx et al. (Fn. 37); The Royal Society/Royal Academy of Engineering (Fn. 44); Friends of the Earth, Negatonnes - An initial assessment of the potential for negative emission techniques to contribute safely and fairly to meeting carbon budgets in the 21st century, 2011.

64) Fuss et al. (Fn. 48), 31; NAS (Fn. 19), S. 7; IPCC, Global warming of $1.5^{\circ} \mathrm{C}$ : Summary for policymakers, 2018, S. 17; Friends of the Earth (Fn. 62), S. 17.

65) Fuss et al. (Fn. 48), 14, IPCC (Fn. 59), S. 17; Minx et al. (Fn. 37), 8; NAS, (Fn. 19), S. 6

66) Fuss et al. (Fn. 48), 32; Der IPCC 2018-Bericht verweist auch auf die Studie von Fuss et al. (2018). Siehe IPCC (Fn. 5), S. 345; NAS (Fn. 19), S. 6, Friends of the Earth (Fn. 62), S. 21.
Tab. 2 Derzeit geschätztes CO2-Entnahmepotenzial für das Jahr $2050^{63}$

\begin{tabular}{|c|c|}
\hline NET & Entnahmepotenzial \\
\hline BECCS & $0,5-10 \mathrm{Gt} \mathrm{CO}$ pro Jahr $^{64}$ \\
\hline Aufforstung/Wiederaufforstung & $0,5-3,6 \mathrm{Gt} \mathrm{CO}$, pro Jahr ${ }^{65}$ \\
\hline $\begin{array}{l}\text { Landmanagement zur Erhöhung } \\
\text { der Kohlenstoffbindung }\end{array}$ & $2-6,9 \mathrm{Gt} \mathrm{CO}_{2}$ pro Jahr ${ }^{66}$ \\
\hline Biokohle & $0,5-4,8 \mathrm{Gt} \mathrm{CO}$ pro Jahr $^{67}$ \\
\hline Beschleunigte Verwitterung & $2-5 \mathrm{Gt} \mathrm{CO}_{2}$ pro Jahr ${ }^{68}$ \\
\hline DACCS & $0.5-5 \mathrm{Gt} \mathrm{CO}_{2}$ pro Jahr ${ }^{69}$ \\
\hline Blue Carbon $\star \star$ & $0,02-0,08 \mathrm{Gt} \mathrm{CO}_{2}$ pro $\mathrm{Jahr}^{70}$ \\
\hline Ozeandüngung & $0.2-3,7 \mathrm{Gt} \mathrm{CO}_{2}$ pro $\mathrm{Jahr}^{71}$ \\
\hline Alkalisierung der Ozeane & $0.99 \mathrm{Gt} \mathrm{CO} \mathrm{CO}_{2}$ pro Jahr ${ }^{72}$ \\
\hline
\end{tabular}

* Einschließlich einer verbesserten landwirtschaftlichen Praxis und der Wiedervernässung von Mooren (letztere ca. 0,37 $\mathrm{GT} \mathrm{CO}_{2}$ pro Jahr) ${ }^{73}$

$\star \star$ Einschließlich Seegraswiesen, Salzwiesen, Mangrovenwälder

kraftausbaus. ${ }^{74}$ Derzeit genießt auch CCS in Deutschland (und wohl auch in weiten Teilen Europas) kein öffentliches und politisches Vertrauen. ${ }^{75}$

Erschwert wird der Einsatz einiger NETs dadurch, dass im Hinblick auf ihre Verwirklichung internationaler Koordinierungsbedarf besteht. Gerade die systematische Entwicklung und dauerhafte Umsetzung naturbasierter NETs erfordert eine funktionierende internationale Koordination, z. B. hinsichtlich der Erhaltung und Nutzung grenzüberschreitender Ökosysteme (Wälder, Seegraswiesen) oder internationaler Räume (Teile der hohen See; Meeresböden, Antarktis) oder der Gewährleistung des Nachschubs bestimmter Güter durch freien Handel (z.B. mit Energiepflanzen). Eine derartige Koordination setzt ein Mindestmaß an politischer und ökonomischer Stabilität in den internationalen Beziehungen voraus. Angesichts aktueller isolationistischer und anti-multilateraler Bestrebungen wichtiger Staaten, scheint es zumindest nicht ausgeschlossen, dass derartige Bedingungen nicht über mehrere Dekaden und ggf. Jahrhunderte verlässlich garantiert wer-

67) Fuss et al. (Fn. 48), 32; Pires (Fn. 40), 511; The Royal Society/Royal Academy of Engineering (Fn. 44), S. 35.

68) Fuss et al. (Fn. 48), 32; Friends of the Earth (Fn. 62), S. 12. Minx et al. verweisen auch auf die Friends of the Earth-Berichte, Minx et al. (Fn. 37).

69) Fuss et al. (Fn. 48), 30.

70) NAS (Fn. 19), S. 62.

71) NAS, Climate intervention - Carbon dioxide removal and reliable sequestration, 2015, S. 60f.; Minx et al. (Fn. 37), 9; EASAC (Fn. 10), S. 27; Friends of the Earth (Fn. 62), S. 19.

72) Enthält nur die von Minx et al. angegebenen Daten für die ocean liming, Minx et al. (Fn. 37). Ocean liming (Meereskalkung) ist der Prozess, bei dem sich gelöster Kalk (slaked lime) im Meer auflöst. Siehe Caserini et al., Affordable $\mathrm{CO}_{2}$ negative emission through hydrogen from biomass, ocean liming, and $\mathrm{CO}_{2}$ storage, Mitig. Adapt. Strateg. Glob. Chang. 2019, 1231, 1234. Laut The Royal Society und Royal Academy of Engineering kann das Minderungspotenzial der Alkalinität der Ozeane bis 2100 bis zu 3500 GT CO betragen. Siehe The Royal Society/Royal Academy of Engineering (Fn. 44), S. 57.

73) International Union for Conservation of Nature (IUCN), Peatlands and climate change, 2017, S. 1.

74) Grundlegend hierzu Peters, Legitimation durch Öffentlichkeitsbeteiligung? Die Öffentlichkeitsbeteiligung am Verwaltungsverfahren unter dem Einfluss internationalen und europäischen Rechts, 2020, i.E.

75) BT-Drs. 19/6891, S. 49. Informativ hierzu Kern et al., The Political Economy of Carbon Capture and Storage: An Analysis of Two Demonstration Projects, Technol. Forecast. Soc. Chang. 2016, 250. 
den können. Hinsichtlich der ökosystemischen Grundlagen gilt es zu bedenken, dass die naturbasierte Entnahme von $\mathrm{CO}_{2}$ durch Schutz, Pflege und ggf. Ausweitung bestimmter Naturräume dauerhaft gewährleistet werden muss. Naturräume sind indes anfällig für Veränderungen und Belastungen, natürliche oder menschengemachte (z.B. Nutzungswandel aufgrund ökonomischer Interessen). Derartige Veränderungen und Belastungen haben sich in den letzten Dekaden trotz aller Schutzbemühungen kontinuierlich verstärkt. ${ }^{76}$ Insbesondere wird erkennbar, dass die derzeitige Abholzung von Wäldern sowie ihre Belastung durch die klimawandelbedingte Zunahme von Trockenperioden weiter voranschreiten. ${ }^{77}$ Dies lässt Maßnahmen wie eine großskalige Aufforstung- und Wiederaufforstung sowie BECCS in manchen Regionen der Welt in naher Zukunft unrealistisch erscheinen.

\subsection{Zur Charakteristik von NETs:}

Mögliche Nebenwirkungen und strukturelle Risiken

Der Einsatz der verschiedenen NETs kann sehr unterschiedliche Nebeneffekte hervorbringen. So wird beispielsweise hinsichtlich BECCS oder hinsichtlich großangelegter Aufforstungs- und Wiederaufforstungsprojekte auf einen damit voraussichtlich einhergehenden wachsenden Bedarf an Land, Wasser und Dünger hingewiesen. ${ }^{78}$ Durch die erheblich erweiterte Nutzung von Anbauflächen kann der Nutzungsdruck auf fruchtbare Böden, Wälder und andere bedeutsame Ökosysteme insgesamt erhöht werden. ${ }^{79}$ Vor allem wird befürchtet, dass ein wachsender Bedarf an Landflächen die global ohnehin stark zunehmende Konkurrenz um Ackerbauflächen weiter verstärkt und sich im schlimmsten Fall negativ auf die Nahrungsmittelsicherheit in ärmeren Ländern auswirkt. ${ }^{80}$

Hinsichtlich eines großskaligen Einsatzes von Biokohle wird ein damit möglicherweise einhergehender Eintrag von Schadstoffen angemahnt (z.B. Schwermetalle oder Pestizide). ${ }^{81}$ Im Rahmen des Verfahrens der beschleunigten Verwitterung könnte es u.a. zu erheblichen physikalischen und chemischen Effekten in Böden sowie in Grund- und Oberflächengewässern kommen. ${ }^{82}$ Der effektive Einsatz dieser NET würde auch den Aufbau riesiger Infrastrukturen erfordern. ${ }^{83}$ Mit Blick auf DAC-Technologien wird wiederkehrend betont, dass die dazu nötigen Anlagen relativ groß sein müssten (also einen erheblichen Materialumsatz erfordern), die Kohlenstoffbindung große Mengen an Energie verbrauchen würde (in Form von Wärme und/ oder elektrischer Energie) und sie den permanenten Einsatz von Trennmitteln voraussetzen. ${ }^{84}$ Bezüglich der CCSTechnologie wird insbesondere das Entweichen größerer Mengen von eingelagertem $\mathrm{CO}_{2}$ befürchtet. Käme es dazu, wären etwaig erzielte positive Klimawirkungen nichtig. Vor allem hinsichtlich der langfristigen Speicherkapazität und -sicherheit bestehe noch Forschungsbedarf. ${ }^{85}$

Nichtintendierte Effekte können auch struktureller Natur sein. ${ }^{86}$ So wird insbesondere befürchtet, dass die Aussicht auf alternative Klimaschutzinstrumente die Anreize zu kostspieligen Mitigationsbemühungen der Staaten weiter sinken lässt (moral hazard). Zum einen könne die Wahrnehmung entstehen, der Bedarf nach ambitionierter Vermeidung sei doch nicht so dringlich (,,man habe noch mehr Zeit"), zum anderen, dass man zwischen zwei gleichwertigen Optionen wählen könne. Die Eröffnung eines derartigen Korridors der Möglichkeiten rufe auch wirtschaftliche Interessen auf den Plan, die von einer Fortführung der Nutzung fossiler Energieträger profitieren. ${ }^{87}$ Das zweite Argument wurde bereits eingangs genannt. Es besteht die Möglichkeit, dass der Einsatz von NETs zu einer zeitlichen und räumlichen Verlagerung des Klimaproblems führt. Im ungünstigsten Fall werden neue Technologien und Verfahren entwickelt, die mit hohem Energieaufwand neue Umweltbelastungen erzeugen, bei insgesamt unsicherem Erfolg.
Jedoch sind bei einigen Maßnahmen durchaus auch positive Nebenwirkungen vorstellbar. ${ }^{88}$ Grundsätzlich mag gelten, dass solange sich die Treibhausgasentnahme und - einlagerung durch eine verbesserte Erhaltung und Nutzung natürlicher Ökosysteme vollzieht oder sie sogar fördert, Synergieeffekte zu anderen Umweltzielen entstehen können. So würde z.B. eine ökologisch orientierte Aufforstung - und Wiederaufforstung, der Anbau und der verbesserte Schutz von Seegraswiesen oder eine ökologischere Landwirtschaft $\mathrm{zu}$ einer Erweiterung und $\mathrm{Zu}-$ standsverbesserung von Naturräumen führen. Im besten Fall würden so Naturräume entstehen oder geschützt und so der Zustand der Umweltmedien und Ökosysteme insgesamt verbessert. ${ }^{89}$ Akzeptierte man weitergehend die Notwendigkeit weiterer Entnahme- und Einlagerungsaktivitäten, sind auch Vorteile anderer Ansätze erkennbar, die insgesamt zur Verbesserung der Treibhausgasbilanz der Atmosphäre beitragen. Beispielhaft wäre hier die Nutzung des durch DAC entnommen $\mathrm{CO}_{2}$ als Treibstoff oder Wertstoff zu nennen (i.S.e. Recyclings fossiler Brennstoffe), durch den die weitere Förderung und Nutzung neuer, d.h. zusätzlicher fossiler Energieträger vermieden werden könnte. ${ }^{90}$

76) Diese Entwicklung vollzieht sich insbesondere seit dem Ende des zweiten Weltkriegs rasant. Siehe Steffen et al., The Trajectory of the Anthropocene: The Great Acceleration, Anthr. Rev. 2015, 81; Steffen et al., Planetary boundaries: guiding human development on a changing planet, Science 2015, 736.

77) Für Deutschland siehe aktuell Bundesministerium für Ernährung und Landwirtschaft, Ergebnisse der Waldzustandserhebung, 2019.

78) The Royal Society/Royal Academy of Engineering (Fn. 44), S. 40.

79) Buck, Rapid Scale-Up of Negative Emission Technologies: Social Barriers and Social Implications, Clim. Chang. 2016, 155, 158.

80) Siehe z. B. Kartha/Dooley, The Risks of Relying on Tomorrow's „Negative Emissions“ to Guide Today's Mitigation Action, Stockholm Environment Institute Working Paper No. 2016-08, 2016, S. 5 ff.; van Vuuren et al., Alternative pathways to the $1.5^{\circ} \mathrm{C}$ target reduce the need for negative emission technologies, Nat. Clim. Chang. 2018, 391. Der Nutzungsdruck auf die dortigen Landgebiete und die Folgen für die Nahrungsmittelsicherheit sind in den letzten zwei Dekaden vor allem in Folge großskaliger Investitionen in Agrarflächen sichtbar geworden. Siehe hierzu Markus, Regulating Large-Scale Farmland Investment in Low Income Countries (,Land Grabbing'): Appraising Different Modes of Transnational Governance, in: Ginzky et al., International Yearbook of Soil Law and Policy, 2020, i.E.; The Royal Society/ Royal Academy of Engineering (Fn. 44), S. 26; Hohlwegler, Moral Conflicts of several „Green“ terrestrial Negative Emission Technologies regarding the Human Right to Adequate Food - A Review, Adv. Geosci. 2019, 39.

81) Teichmann/Kemfert, Biokohle in der Landwirtschaft als Klimaretter?, 2014, S. 4; Fuss et al. (Fn. 48), 25.

82) Fuss et al. (Fn. 48), 22

83) Wiliamson, (Fn. 73), $153 \mathrm{f}$.

84) The Royal Society/Royal Academy of Engineering (Fn. 44), S. 59; Daggash et al., The Role and Value of Negative Emissions Technologies in Decarbonising the UK Energy System, Int. J. Greenh. Gas Control 2019, 181, 184; Fuss et al. (Fn. 48), 17. Hier gilt es sicherlich hinsichtlich verschiedener Anlagen zu differenzieren, siehe Dittmeyer et al. (Fn. 54).

85) Siehe hierzu die derzeitige Einschätzung der Bundesregierung in BT- Drs. 19/6891, S. 14-15.

86) Siehe hierzu auch den Überblick in Schäfer et al., The European Transdiciplinary Assessment of Climate Engineering (EuTRACE: Removing Greenhouse Gases from the Atmosphere and Reflecting Sunlight away from Earth, 2015.

87) Nicht umsonst erforschen vor allem die Staaten die CCS-Technologie, die derzeit primär von der Nutzung fossiler Brennstoffe profitieren. Siehe $S R U$ (Fn. 20), S. 64. Siehe auch Ginzky/Ruddigkeit (Fn. 25).

88) Siehe IPCC (Fn. 5), Abschnitt C. 3.5

89) Zu den positiven Effekten siehe z. B. Fuss et al. (Fn. 48), 25; Macreadie et al. (Fn. 51); Vierros (Fn. 51); Fargione et al. (Fn. 50).

90) Dittmeyer et al. (Fn. 54). 


\subsection{Klimapolitik durch NETs:}

Abschwächung (Mitigation), Anpassung oder Climate Engineering?

Viele der hier angesprochenen Ansätze zur Erzeugung negativer Emissionen werden bereits seit einer ganzen Weile erforscht und einzelne Maßnahmen wurden und werden aktuell zum Zwecke des Klimaschutzes eingesetzt (z. B. die Aufforstung und Wiederaufforstung). ${ }^{91}$ Die Forschungszusammenhänge, die politischen Rahmenbedingungen sowie die Begrifflichkeiten haben sich über die letzten beiden Dekaden aber verändert. Es gilt daher, die Negativemissionstechnologien in den bisher gängigen Begriffsrahmen einzuordnen, um sie dann klarer als bisher von Maßnahmen abzugrenzen, die bereits die Entstehung von Treibhausgasen verhindern sowie von Maßnahmen des sog. SRM.

Einen erheblichen Aufmerksamkeitsschub erhielten Verfahren zur Entnahme von Treibhausgasen aus der Atmosphäre vor gut zehn Jahren. Verwendet wurde vor allem der Begriff Carbon Dioxide Removal (CDR). Damals führte eine deutsch-indische Forscher^innengruppe Eisendüngungsexperimente im Südatlantik durch. ${ }^{92}$ Die Folge war eine öffentliche, politische und rechtliche Debatte um das Thema Climate Engineering (oder Geoengineering), die bis heute nicht ganz abgeebbt ist. ${ }^{33}$ Unter der Überschrift Climate Engineering bzw. Geoengineering wurden auch viele der hier aufgegriffenen NETs diskutiert ${ }^{94}$; darüber hinaus aber auch Maßnahmen des sogenannten SRM, also Methoden die in großem Umfang in den Strahlungshaushalt der Erde eingreifen. ${ }^{95}$ Beide Ansätze verbindet vor allem die Intensität der Eingriffe in Naturabläufe zum Zwecke der Klimamodifikation. ${ }^{96}$ Aufgrund dieser strukturellen Gemeinsamkeit zwischen NETs (einschließlich CDR) und SRM halten einige Autor ${ }^{\star}$ innen an dem Oberbegriff des Climate Engineering bzw. Geoengineering für NETs und SRM fest. ${ }^{97}$

Nach Abschluss des PÜ sowie verstärkt nach Veröffentlichung des ,IPCC-Sonderberichts $1,5^{\circ} \mathrm{C}$ globale Erwärmung" Ende 2018 gibt es den Versuch, NETs aus dieser Begriffsklammer zu lösen und sie als Abschwächungsmaßnahmen (Mitigation) im Sinne des internationalen Klimaregimes $\mathrm{zu}$ interpretieren. ${ }^{98} \mathrm{Nach}$ der KRK erfolgen Maßnahmen zur „Abschwächung der Klimaänderungen“ entweder durch die „Bekämpfung oder „Begrenzung“ anthropogener Emissionen von „Treibhausgasen aus Quellen oder den „Abbau solcher Gase durch Senken“.99 Quelle wird definiert als ein „Vorgang oder eine Tätigkeit, durch die ein Traibhausgas, ein Aerosol oder eine Vorläufersubstanz in die Atmosphäre freigesetzt wird." ${ }^{100}$ Senke wird bezeichnet als ,Vorgang, eine Tätigkeit oder einen Mechanismus, durch die ein Treibhausgas (...) aus der Atmosphäre entfernt wird." ${ }^{101}$ Die Abschwächung (Mitigation) umfasst also einerseits die Bekämpfung oder Begrenzung der Freisetzung von Treibhausgasen in die Atmosphäre, andererseits Vorgänge, Tätigkeiten oder Mechanismen, mit denen Treibhausgase aus der Atmosphäre entnommen werden. ${ }^{102}$

Die Kategorisierung von NETs als Maßnahmen der Abschwächung (Mitigation) einerseits und des Climate Engineering andererseits scheint vor dem Hintergrund ihrer inhaltlichen Offenheit bzw.- Unbestimmtheit grundsätzlich nachvollziehbar. Sobald NETs als Instrumente der Klimapolitik allerdings wirklich an Bedeutung gewinnen, gilt es, diese Subsumtion grundlegend zu überdenken. Die zunehmende Relevanz sowie die Unterschiede zwischen NETs und anderen Maßnahmen der Abschwächung (Mitigation) und des Climate Engineering lassen eine einheitliche begriffliche, analytische und rechtliche Bewertung undifferenziert und damit ungerechtfertigt erscheinen. Entscheidend ist vor allem, dass NETs, würden sie in dem vom IPCC anvisierten Umfang realisiert werden, in ihren Dimensionen und ihren möglichen Nebenwirkungen über alle dagewesenen Ansätze zur Entnahme von Treibhausgasen aus der Atmosphäre hinausgehen würden. Darüber hinaus unterscheiden sich NETs sich aufgrund ihrer Funktionsweise er- heblich von Maßnahmen, die auf die Vermeidung der Entstehung oder der Freisetzung von Treibhausgasen gerichtet sind sowie von Eingriffen in den solaren Strahlungshaushalt der Erde (SRM). ${ }^{103}$ Dabei erweist sich auch der Begriff der „Negativemission“ selbst als präzisionsbedürftig, da nicht jede NET tatsächlich bereits für sich genommen $\mathrm{zu}$ einer effektiven Minderung an Treibhausgasen in der Atmosphäre beitragen kann. Das Begriffssystem bedarf insoweit einer schärfenden Neuordnung.

An dieser Stelle wird daher für eine fünfgliedrige Unterscheidung plädiert, die klarer zwischen Maßnahmen unterscheidet, die 1) die (chemische) Entstehung von Treibhausgasen, 2) ihre Freisetzung in die Atmosphäre, 3) die dortige Akkumulation adressieren, sowie 4) Maßnahmen, die den planetaren Strahlungshaushalt bei gegebener THGKonzentration zu beeinflussen suchen, und 5) solchen, die die negativen Folgen eines globalen Temperaturanstieges abmildern wollen.

Die Gruppen von Maßnahmen variieren grundlegend hinsichtlich

- ihrer Funktionsweise,

- ihrer jeweiligen Ansatzpunkte im Wirkungsgefüge des anthropogen verursachten Treibhauseffekts,

- ihres Beitrags zur Verwirklichung der Klimaschutzziele (insbesondere im Hinblick auf Art. 2 KRK: ,Stabilisierung der Treibhausgaskonzentration in der Atmosphäre") sowie

- hinsichtlich ihrer Nebenwirkungen auf die Natur.

Zur Veranschaulichung soll hier der jeweilige Ansatzpunkt der unterschiedlichen Klimaschutzmaßnahmen in der Wirkungskette des Treibhauseffekts schematisch dargestellt werden.

In der bisherigen Terminologie des Klimaregimes gelten Maßnahmen, die die Bildung, Freisetzung und die Akkumulation von Treibhausgasen adressieren, als Maßnahmen der Abschwächung (Mitigation). Innerhalb dieser Gruppe der Abschwächungsmaßnahmen wurde zwischen solchen

91) Siehe hierzu Fleming, Fixing the Sky: The Checkered History of Weather and Climate Control, 2010.

92) Siehe Gawel, Climate Engineering als Mittel der Klimapolitik, ZUR 2011, 451; Schlacke/Markus/Much, Rechtliche Steuerungsmöglichkeiten für die experimentelle Erforschung der Meeresdüngung, UBA-Texte 20/2012, 2012; The Royal Society, Geoengineering the Climate: Science, Governance and Uncertainty, 2009; Umweltbundesamt, Geo-Engineering - Wirksamer Klimaschutz oder Größenwahn?, 2011.

93) Siehe vor allem den Bericht des Schwerpunktprogramms 1689 der Deutschen Forschungsgemeinschaft (DFG), Climate Engineering und unsere Klimaziele - eine überfällige Debatte, 2019; Krüger, Geoengineering im Völkerrecht, 2020; Armeni/Redgwell, International Legal and Regulatory Issues of Climate Geoengineering Goverance: Rethinking the Approach, 2015. Siehe zur politischen Debatte z. B. BT-Drs. 19/20183.

94) Siehe Überblick in Schwerpunktprogramm der Deutschen Forschungsgemeinschaft 1689, Climate Engineering und unsere Klimaziele - eine überfällige Debatte, 2019, S. $27 \mathrm{f}$.

95) Zum SRM siehe z. B. Moreno-Cruz/Keith, Climate Policy under Uncertainty: A Case for Solar Geoengineering, Clim. Chang. 2013, 431, $431 \mathrm{f}$.

96) Siehe hierzu DFG (Fn. 94), S. 23.

97) Ginzky/Ruddigkeit (Fn. 25).

98) DFG (Fn. 94), S. 24-25.

99) Siehe z.B. Art. 4 Abs. 1 lit. b, Abs. 2 lit. a KRK.

100) Art. 1 Nr. 9 KRK.

101) Art. 1 Nr. 8 KRK.

102) Etwas unklarer ist die Definition des IPCC. Dieser versteht Mitigation als eine ,human intervention to reduce emissions or enhance the sinks of greenhouse gases“. Siehe IPCC (Fn. 5), S. 554.

103) Die Entwicklung und der mögliche Einsatz von Maßnahmen des Solar Radiation Management soll hier nicht diskutiert werden. Siehe hierzu Winter, Klima-Engineering: last exit or exitus, ZUR 2011, 458. 
Abb. 1 Ansatzpunkte von Klimaschutzmaßnahmen in der Wirkungskette des Treibhauseffekts

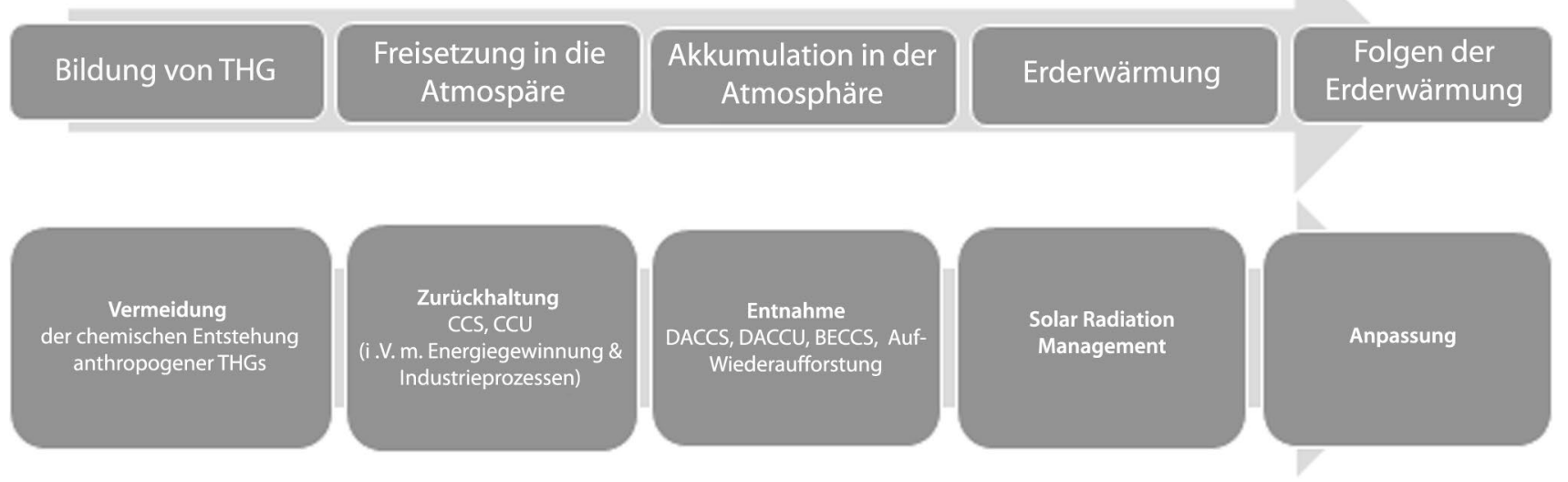

Quelle: Eigene Darstellung

differenziert, die die Freisetzung oder die Akkumulation von Treibhausgasen in der Atmosphäre adressieren. Der Bedeutungszuwachs von NETs und CCS (und ggf. CCU) ändert die Sachlage und erfordert eine andere Differenzierung.

Erstens: Maßnahmen, die auf die Vermeidung der (chemischen) Entstehung von Treibhausgasen gerichtet sind. Sie führen zu einem Unterlassen von Tätigkeiten, bei denen sich anthropogene Treibhausgase bilden, die potenziell in die Atmosphäre freigesetzt werden können. Sie leisten damit einen Beitrag zur Stabilisierung der THG-Konzentration in der Atmosphäre und damit zur Verwirklichung des zentralen Ziels der KRK. Im Zuge der Begrenzung von Treibhausgasen durch Vermeidung der Entstehung kann es zu sogenannten Verlagerungseffekten und innerökologischen Konflikten kommen, weil der Einsatz alternativer Technologien und Verfahren zur Energie- oder Produkterzeugung möglicherweise andere Teile der Umwelt belastet (z.B. Ausbau der erneuerbaren Energien vs. Naturschutz).

Zweitens: Maßnahmen der Zurückhaltung (Retention) setzen nach der Entstehung von Treibhausgasen aber vor ihrer Freisetzung in die Atmosphäre an. Bereits vorhandene Treibhausgase werden vor ihrer Freisetzung in die Atmosphäre abgefangen und eingelagert. Maßnahmen der Zurückhaltung adressieren die für die Stabilität der Klimas problematischen Folgen der chemischen Entstehung anthropogener Treibhausgase, indem sie die Anreicherung von Treibhausgasen in der Atmosphäre zu verhindern suchen. Im Rahmen dieser Maßnahmen stellen sich verschiedene Herausforderungen, insbesondere die Frage der Dauerhaftigkeit der Einlagerung von Treibhausgasen, ihre möglichen Nebenwirkungen, ihre politische Realisierbarkeit vor dem Hintergrund politischer Bedenken gegen die Einlagerung etc.

Drittens: Maßnahmen der Entnahme von Treibhausgasen aus der Atmosphäre setzen erst nach ihrer Übergabe an die Atmosphäre (Freisetzung) an. Treibhausgase werden aus der Atmosphäre entnommen, um dann eingelagert zu werden. Wie die Maßnahmen der Zurückhaltung (Retention) adressieren sie die problematischen Folgen von Treibhausgasen, d.h. ihre zu hohe Konzentration in der Atmosphäre. Auch hier stellen sich die Probleme der dauerhaften Einlagerung, der Nebenwirkungen und der Akzeptanz.

Viertens: Maßnahmen des SRM verhindern weder die Entstehung von Treibhausgasen noch erfolgt eine Entnahme aus der Atmosphäre. Sie bezwecken den Eingriff in den Strahlungshaushalt der Erde, um deren globale Mitteltemperatur zu senken. Die Wirkungen großskaliger SRM-Maßnahmen sind bisher noch weitgehend ungeklärt. Befürchtet wird unter anderem aber, dass es bei einem erhöhten Einbringen von Sulfat-Aerosolen in die Stratosphäre zum Abbau von Ozon, der Verdunklung von den Teilen der Erdoberfläche sowie der Veränderung regionaler oder lokaler Klima- und Wetterbedingungen kommen könnte. ${ }^{104}$ Insbesondere ein plötzlicher Abbruch von SRM-Maßnahmen könnte zu einer dramatischen Beschleunigung klimatischer Veränderungen führen.

Fünftens: Anpassungsmaßnahmen vermeiden und entnehmen keine Treibhausgase aus der Atmosphäre. Sie tragen damit selbst nicht zur Stabilisierung der Treibhausgaskonzentration in der Atmosphäre bei. Ihr Ziel ist der bestmögliche Umgang mit den Folgen der erhöhten Treibhausgaskonzentration. ${ }^{105}$

Diese Einteilung macht deutlich, dass NETs Maßnahmen umfasst, die an zwei unterschiedlichen Punkten der Wirkungskette des Klimawandels ansetzen, nämlich Maßnahmen zur Entnahme (removal) bereits an die Atmosphäre übergebener Treibhausgase sowie Maßnahmen der $\mathrm{Zu}$ rückhaltung (Retention). Der Begriff NETs kann also nicht unter den Begriff der Abschwächung (Mitigation) subsumiert werden. Ob Treibhausgase tatsächlich dauerhaft entfernt werden (ob also Negativemissionen entstehen), hängt von weiteren Bedingungen ab, nämlich der Möglichkeit einer dauerhaften Zurückhaltung (Retention).

Dies verdeutlicht auch das Verhältnis der verschiedenen Maßnahmen untereinander. Maßnahmen, die die Entstehung von Treibhausgasen verhindern, implizieren, dass insoweit vermiedene Treibhausgase nicht unter Einsatz von Energie, Technik, oder Eingriffen in die Umwelt im Wege der Entnahme und Einlagerung ,klimaunwirksam“ gemacht werden müssen. Demgegenüber bleibt zu beachten, dass auch die Vermeidung der Entstehung von Treibhausgasen den Einsatz von Energie und Technik sowie Umwelteingriffe erfordern kann (z.B. der Ausbau der Windkraft, Nutzung von Biomasse). Es ist eine empirische Frage, welche volkswirtschaftlichen Kosten (einschließlich der Umweltkosten) jeweils maßnahmenbezogen höher liegen. Zwischen den Maßnahmen der Zurückhaltung und Entnahme einerseits und SRM-Maßnahmen andererseits liegt der Unterschied vor allem darin, dass - zumindest in der Theorie - die Zurückhaltung und Entnahme von Treibhausgasen zur Zielerreichung der KRK beitragen können, SRM dagegen nicht. Dadurch würden sie auch die Umwelt von den Folgen steigender Treibhausgaskonzentrationen in der Atmosphäre entlasten (insbesondere würden sie den Treibhauseffekt und die Versauerung der Ozeane reduzieren). SRM-Maßnahmen wären als Ewigkeitslast dauerhaft aufrechtzuerhalten, da die anthropogenen THG-Quellen nicht

104) DFG (Fn. 94), S. 36-38.

105) Siehe dazu Gawel/Heuson, Ökonomische Grundfragen der Klimaanpassung, Wirtschaftsdienst 2012, 480. 
abgestellt werden. Des Weiteren werfen sie erhebliche globale Governanceprobleme und Naturrisiken auf.

\section{Zwischenergebnis}

Um Treibhausgase aus der Erdatmosphäre zu entnehmen, kommen zahlreiche, in ihrer Charakteristik höchst unterschiedliche Verfahren und Maßnahmen in Betracht, die als „Negativemissionstechnologien“ (NETs) zusammengefasst werden. Ob der Einsatz dieser Verfahren tatsächlich zu Negativemissionen beiträgt, hängt vielfach erst noch von weiteren Bedingungen ab. Eine Subsumtion unter Mitigation ist abzulehnen; NETs stellen eine eigene klimapolitische Maßnahmenklasse dar, die sich von anderen klimapolitischen Ansätzen, aber auch untereinander, durch ihre Funktionsweise, ihren Beitrag zur Verwirklichung der Klimaschutzziele und ihre Nebenwirkungen sowie ihre Governance deutlich unterscheiden. Dies muss sich auch bei der Verortung im Regelwerk des internationalen Klimaregimes angemessen abbilden (dazu Teil 2).
Open Access. Dieser Artikel wird unter der Creative Commons Namensnennung 4.0 International Lizenz veröffentlicht, welche die Nutzung, Vervielfältigung, Bearbeitung, Verbreitung und Wiedergabe in jeglichem Medium und Format erlaubt, sofern Sie den/die ursprünglichen Autor(en) und die Quelle ordnungsgemäß nennen, einen Link zur Creative Commons Lizenz beifügen und angeben, ob Änderungen vorgenommen wurden.

Die in diesem Artikel enthaltenen Bilder und sonstiges Drittmaterial unterliegen ebenfalls der genannten Creative Commons Lizenz, sofern sich aus der Abbildungslegende nichts anderes ergibt. Sofern das betreffende Material nicht unter der genannten Creative Commons Lizenz steht und die betreffende Handlung nicht nach gesetzlichen Vorschriften erlaubt ist, ist für die oben aufgeführten Weiterverwendungen des Materials die Einwilligung des jeweiligen Rechteinhabers einzuholen.

Weitere Details zur Lizenz entnehmen Sie bitte der Lizenzinformation auf http://creativecommons.org/licenses/by/4.0/deed.de.

Open Access funding enabled and organized by Projekt DEAL.

\section{Das Töten von wildernden Hunden als Maßnahme des Jagdschutzes}

\section{Andreas Berg}

\section{(c) Springer-Verlag GmbH Deutschland, ein Teil von Springer Nature 2021.}

„Als Hund eine Katastrophe, als Mensch unersetzlich"1 - diese Bilanz des ehemaligen Bundespräsidenten Johannes Rau wird vielen Hundehaltern nachvollziehbar erscheinen. Nicht angeleint hat sich allerdings schon der treueste Familienhund seines Raubtierrudiments besonnen und verfolgt urplötzlich das vor ihm abspringende Reh. Der Jagdschutz ermächtigt nahezu bundesweit bestimmte Personen, wildernde Hunde erschießen zu dürfen. Es scheint geboten, der Anwendung dieser speziellen Form der Gefahrenabwehr mit gebotener Ambivalenz zu begegnen.

\section{Einleitung}

Dass die Beziehung zwischen Mensch und Hund ${ }^{2}$ in groBen Teilen der Welt von einer Herzlichkeit eigener Art geprägt ist, wurde schon treffend 1518 beschrieben: Der beste Freund des Menschen. ${ }^{3}$ Aber auch Freundschaften unterliegen bekanntermaßen Regeln, die für ein geordnetes Miteinander unerlässlich sind. Der Ablauf eines gemeinsamen Spaziergangs ist dabei sogar gesetzlich konturiert. Mancher Vierbeiner widersetzt sich jedoch hin und wieder eigensinnig der mahnenden Ansprache seiner Aufsichtsperson. Trifft ein freilaufender Familienhund sodann z.B. auf Hase oder Reh, entzieht er sich oftmals dem Einwirkungsbereich des Hundeführers und verliert sich instinktiv in triebgesteuerter Verfolgungsabsicht. ${ }^{4}$ Ursächlich ist zumeist die Unkenntnis bis hin zur Uneinsichtigkeit vieler Hundebesitzer über die vom freilaufenden Hund ausgehende Gefahr. Oft verenden Wildtiere aufgrund erlittener Verletzungen erst einige Zeit später, teils qualvoll. ${ }^{5}$ Die im Fokus stehende Gefahrenab-

\section{Dr. iur. Andreas Berg,}

Lehrbeauftragter für Verwaltungsrecht, Lehrstuhl für Öffentliches Wirtschaftsrecht, Technik- und Umweltrecht von Univ.-Prof. Dr. iur. Rainer J. Schröder, Universität Siegen, Siegen, Deutschland wehrmaßnahme, deren landesjagdrechtliche Ausgestaltungen teils deutlich voneinander abweichen, wirkt dabei unter Berücksichtigung der emotionalen Verbindung zwischen Hund und Herrchen in ihrer Ausübung trotzdem nahezu barbarisch. Zweifellos begründet dies zwischen dem benamten, in einer Familienstruktur eingebundenen Hund und dem herrenlosen Wildtier einen Dualismus von Existenzwertigkeiten. Zum Nachdenken regt an, dass im Jagdjahr 2007/2008 beispielhaft in Nordrhein-Westfalen im Rahmen

1) Johannes Rau über seinen Hund Scooter, in: Der Spiegel, Stand 3.1.2021, abrufbar unter https://www.spiegel.de/panorama/bundespraesident-scooter-warf-rau-um-a-231115.html.

2) Im Jahr 2019 gab es in Deutschland ca. 8,8 Millionen Hundebesitzer, vgl. Statista, Haustierbesitzer in Deutschland nach Anzahl von Hunden im Haushalt von 2016 bis 2019, Stand 3. 1.2021, abrufbar unter https://de.statista.com/statistik/daten/studie/181167/umfrage/haustier-anzahl-hunde-im-haushalt.

3) Erasmus von Rotterdam: „Iam uix ullum animal amicius quam canis, ...", Desiderius Erasmus: Apvd Seb. Gryphivm, 1542, S. 635 Stand 20.7.2020, abrufbar unter http://books.google.com/books ?id=_HdTAAAAcAAJ\&pg=PA635

4) Vgl. OLG Stuttgart, Urt. v. 8.4.1986 - 12 U 259/85, NJW-RR 1986, 1415, 1416; schon vor ca. 100 Jahren hat der Gesetzgeber mit der Gefährdungshaftung in $₫ 833$ BGB dem unberechenbaren tierischen Verhalten Rechnung getragen, dazu: OLG Koblenz, Beschl. v. 18.10.2018 - 1 U 599/18, NJW 2018, 3596; Fichtlmeier beschreibt hundebezogen den Instinkt allgemein als durch einen Reiz ausgelöstes, nicht erlerntes und formstabil-stereotypes Verhaltensmuster, Grunderziehung für Welpen, 2005, S. 12.

5) Beispielhaft Oberbayerisches Volksblatt GmbH \& Co. Medienhaus KG (OVB), Stand 3.1.2021, abrufbar unter https://www.ovb-online.de/rosenheim/chiemgau/hund-reisst-rehe-prien-jaeger-sagttiere-seien-qualvoll-verendet-13174714.html; Münchener ZeitungsVerlags GmbH \& Co KG (Merkur), Stand 3.1.2021, abrufbar unter https://www.merkur.de/lokales/schongau/peiting-freilaufenderhund-reisst-reh-jaeger-filmt-grausame-szenen-zr-12767360.html. 